University of Rhode Island

DigitalCommons@URI

Open Access Master's Theses

2021

\title{
THE RELATIONSHIP BETWEEN ORAL HEALTH DEFICITS AND ORAL HEALTH RELATED QUALITY OF LIFE IN THE OLDER ADULT POPULATION
}

Lisa Proffitt

University of Rhode Island, Iproff68@verizon.net

Follow this and additional works at: https://digitalcommons.uri.edu/theses

\section{Recommended Citation}

Proffitt, Lisa, "THE RELATIONSHIP BETWEEN ORAL HEALTH DEFICITS AND ORAL HEALTH RELATED QUALITY OF LIFE IN THE OLDER ADULT POPULATION" (2021). Open Access Master's Theses. Paper 1938.

https://digitalcommons.uri.edu/theses/1938

This Thesis is brought to you for free and open access by DigitalCommons@URI. It has been accepted for inclusion in Open Access Master's Theses by an authorized administrator of DigitalCommons@URI. For more information, please contact digitalcommons-group@uri.edu. 
THE RELATIONSHIP BETWEEN ORAL HEALTH DEFICITS AND ORAL

HEALTH RELATED QUALITY OF LIFE

IN THE OLDER ADULT POPULATION

BY

LISA PROFFITT

\author{
A THESIS SUBMITTED IN PARTIAL FULFILLMENT OF THE \\ REQUIREMENTS FOR THE DEGREE OF \\ MASTER OF SCIENCE \\ IN \\ HUMAN DEVELOPMENT AND FAMILY SCIENCE \\ DEVELOPMENTAL SCIENCE
}

UNIVERSITY OF RHODE ISLAND

2021 
MASTER OF SCIENCE THESIS

OF

LISA PROFFITT

APPROVED:

Thesis Committee

Major Professor Phillip Clark

Melanie Brasher

Mary Greaney

Brenton DeBoef

DEAN OF THE GRADUATE SCHOOL

UNIVERSITY OF RHODE ISLAND

2021 


\begin{abstract}
Oral comfort plays a vital role in the older adult population in which social events and participation require adequate oral health to avoid pain and discomfort. Oral discomfort may discourage social experiences and decrease an individual's overall quality of life. Evaluating the relationship between one's oral health and quality of life is dependent on a variety of influences, including oral health deficits, such as using a denture appliance or having inadequate number of teeth to function and maintain oral comfort. The current study aims to examine the relationship between one's oral health status and Oral Health Related Quality of Life (OHRQoL) in the older adult population, and to explore if poorer oral health contributes to reducing OHRQoL. The current analysis uses data from the 2008 wave of the National Health and Nutrition Survey (NHANES) obtained via the Inter-University Consortium for Political and Social Research (ICPSR) website. Using specific variables in the nutritional survey, a composite score was created to represent individual oral healthrelated quality of life (OHRQoL) scores for respondents 60+ years old. The newly created composite score was reliable $(\alpha=0.823)$. A linear regression was conducted to evaluate the relationship between oral health deficits and OHRQoL scores. The results indicated that there was a significant relationship between oral health deficits and OHRQoL scores $(\mathrm{p}<0.001)$. Having posterior teeth versus no posterior teeth had the strongest contribution to OHRQoL scores. Respondents with 20+ teeth present had increased OHRQoL compared to those who had less than sixteen teeth present which decreased OHRQoL. The second linear regression model added controls for demographic factors. The results indicated that both education and race/ethnicity were
\end{abstract}


associated with OHRQoL scores $(\mathrm{p}<0.001)$. These results indicate that both demographic predictors, as well as oral health status, are correlated with OHRQoL scores. The negative effects of poor oral health in the older adult population require

further investigation and should be considered a public health priority to seek ways to improve oral health in this growing population, and to reduce the negative influence these disparities have on healthy living in the older adult population. 


\section{ACKNOWLEDGMENTS}

I would like to thank and extend my enormous appreciation to several people for their wisdom, encouragement, and guidance through my journey here at the University of Rhode Island. First and foremost, I would like to thank my major advisor, Dr. Phil Clark, for pushing me to do my best work. And, for the many suggestions and invaluable insight you have provided throughout many of my courses, my internship, and, of course, my thesis work. Phil, I am forever grateful for your wisdom and style of delivery. Additionally, I would like to thank Dr. Melanie Brasher who has given me invaluable insight and knowledge from the very start of my thesis project. Dr. Brasher has assisted me with the development of a hypothesis, and all the way to the finish line- I am forever grateful. Dr. Mary Greaney, thank you for your valuable suggestions and insight, you have been a great resource through my thesis writing process.

I would also like to thank Dr. Steven Peiser and Libby. Thank you for traveling with me on so many of my life adventures and most importantly, this one. Finally, I would like to thank my mom and dad for always making me believe that anything is possible. Thank you for always holding me- "Trophy High!"

- How far you go in life depends on your being tender with the young, compassionate with the aged, sympathetic with the striving, and tolerant of the weak and strong. Because someday in your life you will have been all of these 


\section{TABLE OF CONTENTS}

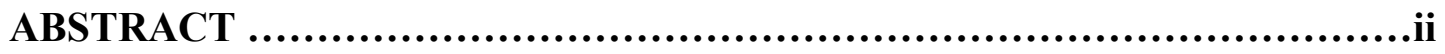

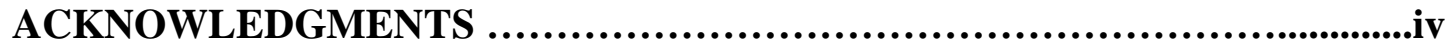

TABLE OF CONTENTS .................................................................v

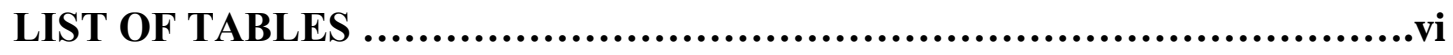

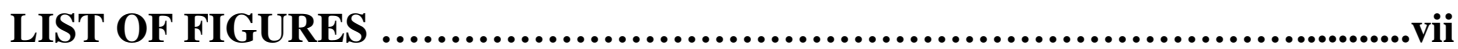

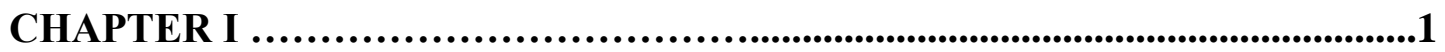

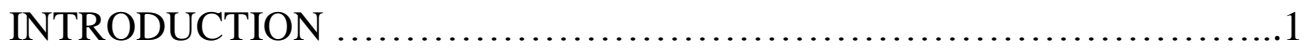

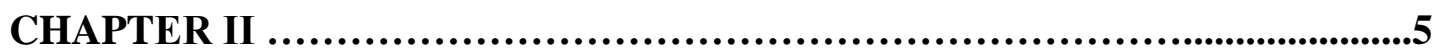

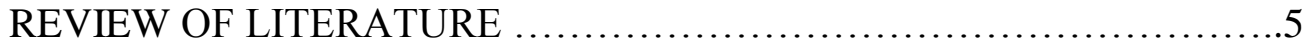

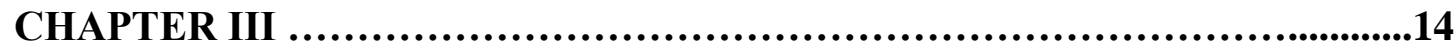

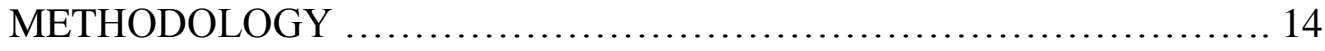

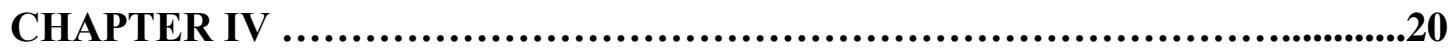

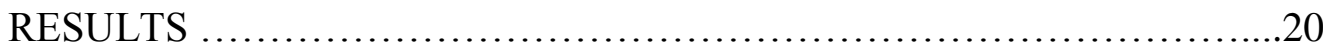

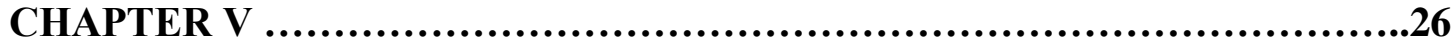

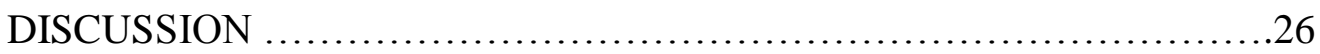

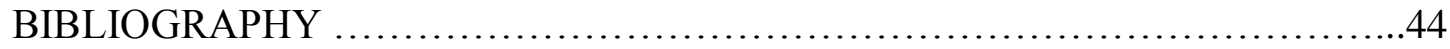




\section{LIST OF TABLES}

TABLE $\quad$ PAGE

Appendix A- Glossary of Terms............................................. 37

Appendix B-NHANES Survey Questions via Interview......................39

Table 1- Independent Variable Attribute...................................43

Table 2- Frequency Dependent Variable..................................21

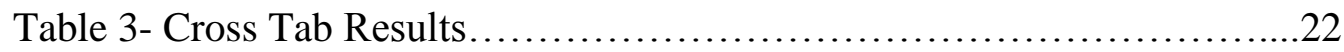

Table 4- Linear Regression Results......................................24

Table 5- Mean OHRQoL scores per Oral Health Status .........................25 


\section{LIST OF FIGURES}

FIGURES

Denture Apparatus Illustration-Figures-1a \& 1b......................... 41

Illustration for IV Attributes (contacted teeth)-Figures 2a \& 2b..............42 


\section{CHAPTER I}

\section{INTRODUCTION}

"Quality of life for people in their seventh decade of life and beyond is dependent on the status of one's mind, mobility and mouth"

\section{-S.J. Peiser}

Research has suggested that one of the keys to healthy aging is related to social involvement, being connected to society, and engaging in social activities such as dining with friends and maintaining and enjoying healthy eating habits. However, one needs to have adequate oral health in order to enjoy these activities of social dining, eating in general, and having unrestricted food choices to maintain healthy eating behaviors (Bennadi \& Reddy, 2013). The concept of Quality of Life (QoL) for the older adult population includes these social activities, in addition to maintaining mental and overall well-being according to the World Health Organization (WHO). WHO further established Oral Health Related Quality of Life (OHRQoL) as an extension of the framework of QoL. The dimensions of OHRQoL include: an individual's comfort while engaging in eating, oral function, speaking, sleeping, and oral comfort overall (Sischo \& Broder, 2011). More recently, there is evidence to support that decline in one's oral health may impact food choices, daily oral comfort, and ultimately OHRQoL. Furthermore, poor oral health may lead to increased discomfort while engaging in social activities, such as social dining and the consumption of food, that may lead to food avoidance and ultimately reduce OHRQoL (Bennadi \& Reddy, 2013). Finally, the biopsychosocial model associated with oral 
health and determinants of social involvement is important in the aging population to promote inclusion and healthy living (Sischo \& Broder, 2011). Successful aging is dependent on the biopsychosocial connection that includes, maintaining optimal health to facilitate social connections which is a determinant of emotional well-being in the older adult population (Moody \& Sasser, 2021). OHRQoL is a dimension included in the biopsychosocial relationship, and OHRQoL measures may be a predictor encouraging or discouraging social outcomes and ultimately influence an individual's emotional well-being (Douglas, Georgiou, Westbrook, 2017).

The focus of my research is on examining the relationship between oral health and OHRQoL. This study will examine the hypothesis that individuals with poorer oral health will also have lower OHRQoL. Additionally, the study will indirectly examine the contribution of food restrictions as a component influencing OHRQoL due to the status of one's oral comfort and function. Finally, this study will further examine the types of oral appliances used, if any, required in restoring oral function, and if such appliances are an adequate substitute for natural teeth in order to restore one's habitual oral function. Several studies have shown that oral comfort may dictate food choices and thereby influence OHRQoL in older adults, and such food selections are generally determined as a result of a combination of oral health deficits, including: missing teeth, partial denture or full denture use, medication-induced xerostomia, and oral dysphagia (Appendix A), as well as poor oral muscle strength (Emami, Kabawat, \& Feine, 2013; John et al., 2016). Therefore, food modification or avoidance may decrease one's OHRQoL, as well as, influence social engagements and social activities, such as dining with others. 
According to current US statistics, the number of people $65+$ years of age will double by 2060 (Hummel, Wilson, Marker, \& Nunn, 2002). Studies have shown that this particular population will have fewer natural teeth and may rely on tooth replacement techniques, such as full dentures or partial dentures, to rehabilitate oral function (Walls \& Steele, 2004). Additionally, tooth loss is significant in the seventh decade of life. However, the process of aging by itself is not a risk factor for tooth loss (Wakabayashi et al., 2016). Studies have shown that poor oral health, including tooth loss, is a contributing risk factor that may influence food choices in the older population (Savoca et al. 2009). Therefore, denture replacement therapy has been routinely utilized to restore occlusion and facilitate adequate nutritional intake (Wakabayashi et al., 2010). However, full and partial denture use has significant limitations, which may affect one's chewing and swallowing abilities due to the instability and lack of comfort the oral appliance provides, which may restrict or limit heathy eating habits (Wakabayashi et al., 2010). Furthermore, having twenty natural teeth or ten opposing pairs of natural teeth is considered optimal for proper oral function, comfort, and mastication of food (Gotfredsen, Walls, Angus, 2007; Hummel et. al., 2002). Consequently, the oral health status of an individual may influence behaviors that are not conducive to proper nutrition due to oral deficits, as well as reduce healthy eating habits and hinder daily activities that will contribute to a decreased OHRQoL.

The current study is a secondary analysis of data collected from the National Health and Nutritional Survey (NHANES) 2007-2008 wave using respondents who are $60+$ in age in the community-dwelling population in the US. A composite 
measure was created using items in the NHANES survey questionnaire to represent OHRQoL scores. Currently, the General Oral Health Assessment Index (GOHAI) is a universal instrument used to measure individual OHRQoL scores (John et al., 2016). The GOHAI metric is proven to be a valid and reliable instrument to measure OHRQoL outcomes (John et al., 2016). Furthermore, research has indicated that survey questionnaires are an adequate source to determine OHRQoL (Bennadi \& Reddy, 2013). The present study created a composite index using items from the NHANES survey that will serve to mimic or replicate similar items used in the GOHAI instrument. The present study will not be directly using the GOHAI instrument, because the specific instrument items are not available in the NHANES oral health survey. Therefore, the current study selected six items from the selfreported oral health questionnaire section of the dietary survey that will represent OHRQoL scores unique to this study, using a 5-point Likert scale. An index of oral comfort and function was created by calculating the mean score for respondents who have three or more non-missing items. Construct validity was assessed using Cronbach Alpha (0.823). Multivariate regression was conducted to compare OHRQoL measures unique to this study with the Independent Variable (IV) containing four mutually exclusive attributes that represent the associated oral health status for each respondent. 


\section{CHAPTER II}

\section{REVIEW OF LITERATURE}

\section{Oral Health Deficits and Dietary Restrictions}

An individual's general health can be influenced by the status of their oral health, and the status of one's oral health may impact the quality of daily oral function, including chewing and eating, that will ultimately determine one's food choices (Spanemburg, Cardoso, Slob, \& Lopez, 2019). Studies confirm that one's perception of their oral health is just as important as clinical findings and can impact daily decisions such as food choices (Spanemburg et al., 2019). One's perception of their oral health status is included as a measure in a model known as Oral Health Related Quality of Life (OHRQoL) (Spanemburg et al., 2019). QHRQoL is defined as a multifactorial scale that includes a person's comfort when eating, sleeping, and engaging in social activities, as well as oral health satisfaction (Bennadi \& Reddy, 2013). Therefore, OHRQoL reflects the full scale of oral health issues and may be a strong predictor of the oral health and food selection relationship (Cousson et al., 2011; Wu, Cheung, Lam, \& Gao, 2018). Finally, the status of an individual's oral health may influence food choices, daily activities, and possibly avoidance of these activities due to oral discomfort and lack of function that will impact social outcomes (Bennadi and Reddy, 2013).

Unfortunately, many studies have focused on dietary restrictions related to systemic causes and not the oral physiological components of dietary restrictions related to the health of the oral cavity, which is where nutritional intake and the digestive process begin (Kossoni, 2018). Oral health restrictions associated with 
limited food choices, food modifications, and oral discomfort include: xerostomia, impaired swallowing issues, and, more significantly, tooth loss/inadequate functioning dentition (Appendix A), and the use of a denture/partial denture (Cousson et al., 2011). Tooth loss is an important indicator in the oral health status of an individual, and may be a predictor of food choices (Tiwari, Scarbo, Bryant, \& Puma, 2017) Some studies have shown that certain food choices are related to and dependent on one's oral health, in particular the number of functioning teeth present for proper mastication (Appendix A) (Toniazzo, Amorim, Muniz, \& Weidlich, 2018). Also, the number of posterior occluding teeth may be an important influence in maintaining a healthy nutritional status (Toniazzo et al., 2018). Numerous studies report that 20 teeth or 10 occluding pairs of teeth are required for proper function and mastication of food (Gotfredsen et al., 2007). Additionally, tooth loss may impair the chewing ability, taste, and comfort of the normal masticatory process (Griffin, Jones, Brunson, Griffin, \& Bailey, 2012). Furthermore, access to care issues among older adults may inhibit this population from seeking proper oral health care, which may ultimately contribute to tooth loss (Tiwari et al., 2017). Finally, Rodrigues et al.'s (2010) study evaluated older adults and found that tooth loss was the biggest annoyance followed by the use of an ill-fitting denture appliance that determined food selections or food avoidance. Therefore, oral discomfort and functional concerns can contribute to one's daily choices and may restrict one's behavior that will impact OHRQoL (Spanemberg et al., 2019).

Conflicting research suggests that dentures (Appendix A) serve as an adequate replacement for missing teeth; however, denture appliances have functional limitations as well (Walls, Steele, Wu, Cheung, Lam, 2004). Several studies support that denture 
appliances do not serve as a substitute for natural teeth but, rather, are a dissatisfying and unstable replacement (Bassadet et al., 2013: Wilson, 2002). Lucena et al.'s (2011) study reported that $40 \%$ of denture users were dissatisfied with the fit and function of a denture, with a lower denture appliance having greater dissatisfaction than an upper denture appliance (Lucena, Gomes, Silva, Cury, 2011). A similar study by Hummel et al. (2002) reported that people with denture appliances were three times more likely to report overall dissatisfaction with eating than individuals who had natural teeth, and instability and retention issues were reported as the main concern (Hummel, Wilson, Marker, Nunn, 2002). Many older adults report that eating healthy and dining with friends are an important aspect of daily living, and eating is a significant element of social participation (Ferreira, Tura, Siva, \& Ferreira, 2017). Therefore, the emotional implications of an unstable denture appliance may impact social participation, due to poor oral appliance function, and ultimately decrease OHRQol (Prithviraja, Madan, Harshamayi, Kumar, \& Vashisht, 2014). Hence, adequate oral health and a functioning dentition will promote a more positive and beneficial food selection that can facilitate a proper diet, promote socialization, and positively influence OHRQoL. Another modifying oral condition that may be used as an indicator for OHRQoL scores includes xerostomia, which will affect one's daily oral comfort. Xerostomia, also known as dry mouth, is a common condition in older adults and can affect one's oral comfort levels (Quandt et al., 2011). Xerostomia is an indicator used in the GOHAI measure to represent the psychosocial impact of this condition on OHRQoL scores (John et al., 2016). However, aging alone is not associated with dry mouth, but rather it is a symptom related to other causes often associated with the 
older adult population (Quandt et al., 2011). Polypharmacy (Appendix A) has been reported as being a strong contributing cause for dry mouth in $20-40 \%$ of the older adult population, which will influence oral comfort in such individuals (Quandt et al., 2011). Polypharmacy is indicated as an indirect contribution to one's oral comfort due to side effects, although it is not directly used as a measure for oral health or OHRQoL indices. Quandt et al.'s (2011) study reports that dry mouth was associated with selfreported food modifications and avoidance of certain foods. The same study suggests that a dry mouth condition affects one's perceived swallowing ability, and this condition may affect consumption of hard to swallow foods, such as fibers. Additionally, people with dry mouth may avoid foods that are challenging to chew, interfere with denture use, and cause additional oral pain (Quandt et al., 2011). Therefore, there are multiple oral health complications that exist individually and collectively, are associated with oral health-related quality of life (QHRQoL), and ultimately impact daily food choices (Quandt et al., 2011; Wu et al., 2018). Additionally, oral pain and discomfort may be a predictor of one's oral health status and have a significant impact on quality of life for the older adult (Lamster \& Northridge, 2008).

\section{Dental Care Disparities Contributing to Oral Health Status}

There is an indirect relationship between OHRQoL and oral health care disparities, whereby adequate access to dental care is related to higher OHRQoL scores and limited dental care is related to lower OHRQoL (Sischo \& Broder, 2011). Professional dental care alone is not a measure for OHRQoL indices; however, effective dental treatment, or lack thereof, may have a positive or negative 
contribution to one's oral health status. Therefore, effective dental care is essential to maintain optimal oral health and prevent a decline in oral health that will have a positive or negative impact, respectively, on one's OHRQoL scores (Tiwari et al., 2017). Access to dental care to maintain optimal oral health, as well as facilitate general health, is a concern especially among the older adult population.

Unfortunately, there are few studies available examining oral health disparities in older adults; however, the Surgeon General's report argues that older Americans who are affected by oral disease are more likely to be disadvantaged, and individuals below the US poverty line were more likely to be edentulous (Lamster \& Northridge, 2008). Individual groups that have a higher prevalence of living below the US poverty line include: African Americans and Hispanics (Lamster \& Northridge, 2008).

Furthermore, oral disease remains one of the most common chronic untreated conditions in the older adult population (Kum, Northridge, \& Metcalf, 2018). Tooth loss is an important indicator of oral health status, with a multi-factorial foundation including poor diet, financial resources, and lack of access to dental professionals (Tiwari et al., 2017). Also, older adults with untreated dental issues were more likely to report oral pain and food avoidance due to poor oral health. These results were seen more frequently among people 55+ years of age (Griffin et al., 2012). Finally, dental care disparities prevent older adults from seeking proper dental care, and those adults with the highest needs are least likely to seek dental care (Griffin et al., 2017). Thirtyseven per cent of older adults in the US have some type of dental insurance coverage, while the remaining $63 \%$ have no dental coverage, making dental care accessibility challenging to maintain optimal oral health (Elder Care Workgroup Report, 2020). 
Access to dental care issues may have a significant contribution to tooth loss, resulting in poor oral health in the older adult population (Griffin et. al). A study conducted in rural Colorado of older adults $65+$ found that there was a statistically significant relationship between tooth loss and not having dental insurance or access to care (Tiwari et al., 2016). Statistical frequencies in the same study reported $15 \%$ of participants between $65-75$ years, $25 \%$ between $75-85$ years, and $58 \%$ above the age of 85 were edentulous (Tiwari et al., 2016). Edentulism is defined as a condition of being toothless (Emami, DeSousa, Kabawat, \& Feine, 2013). Interestingly, it has been suggested that there are few studies available regarding the prevalence of edentulism, and the analysis of oral disparities rarely provides formal statistics, but is rather "cursory prevalence of this condition" (Wu, Liang, Plaman, Relm, \& Luo, 2014). Additionally, Toniazzo et al.'s (2018) study supports the relationship between poor eating habits and fewer teeth, but no significant relationship between denture wear and eating habits. However, edentulism is considered "the final marker of disease burden in oral health" (Emami, et al., 2013, p. 1), and edentulism directly negatively impacts OHRQoL measures (Bennadi \& Reddy, 2013).

\section{Oral Health Status and Social Involvement Impacting OHRQoL}

Oral health is essential for proper eating, as well as reducing any oral restrictions one may have when engaging in social dining experiences (Douglas, Georgiou, Westbrook et al., 2016). The World Health Organization (WHO) argues that social participation is a major component in healthy aging, and such participation is included in several models determining successful aging in the older adult population (Douglas et al., 2017). Unfortunately, oral health deficits may negatively 
impact social interactions due to pain, poor self-esteem, or poor function which will decrease OHRQoL (Sicho \& Broder, 2011).

Quandt et al.'s (2009) study reports that food choices and modification were significantly related to an individual's self-rated and perceived oral status, and people with limited dentition or dentures chose softer foods that were less challenging to manipulate. Furthermore, individuals avoided foods that caused oral pain due to oral health deficits, including dry mouth issues or ill-fitting dentures (Quandt et al., 2009). Such food modifications often occur gradually over a period of time, and one may not be conscious of their actions to accommodate for oral health deficits (Quandt et al., 2011). Some of the most common foods that are avoided include: fruits and vegetables, meats, nuts, and fiber (Savaco et al., 2010). The avoidance of such foods may indirectly affect social dining experiences based on one's perception of their oral restrictions, limitations, and comfort (Spanemberg et al., 2019).

The relationship between oral health and food choices or food modifications consists of a cascading effect, with each level having an impact on proper food selections. Multifactorial and interrelated oral health conditions impacting food choices include: dry mouth, swallowing difficulty, chewing issues, poor or ill-fitting dentures, and inadequate number of remaining teeth (Emami et al., 2013). Studies have shown that individuals with limited posterior occlusion (Appendix A) or denture use may have difficulty chewing certain foods, and will ultimately choose softer and less challenging foods (Emami et al., 2013). These challenges can be magnified in an individual with oral pain or a dry mouth issue that can create additional swallowing issues for foods already difficult to chew (Emami et al., 2013). Therefore, any oral 
health deficit can lead to food avoidance, with the extent of food avoidance dependent on the severity and number of oral conditions present. Research confirms that individuals with oral health deficits consume a lower quality diet than is required to maintain one's general health, and this may be a consequence of one's decreased OHRQoL (Emami et al., 2013). Finally, individual eating habits, limitations, or restrictions seem to be a strong determinant of whether an individual participates in social experiences, such as dining with others, or sustains social withdrawal behaviors (Douglas et al., 2016). The physical and emotional components required for social participation may be one of the strongest predictors for one's OHRQoL scores with the older adult population (Ferreira, Tura, Da Siva, Ferriera, 2017). This correlation between physical and emotional indicators represents the biopsychosocial relationship with OHRQoL measures (John et al., 2016). Furthermore, it has been suggested that emotional health and social functioning have a strong relationship with oral health conditions (Spanemberg et al., 2019).

\section{Justification of Current Study}

Quality of life for the older adult population involves such dimensions as participation with friends and family members in social dining experiences, and enjoying a variety of food selections. The purpose of the current study is to further examine the determinants of OHRQoL associated with oral health deficits or restrictions that may impact such activities as dining and other social activities. Future direction to improve QoL in the older adult population should consider the impact of oral deficits affecting OHRQoL outcomes. Additionally, US statistics have indicated that the older adult population will double in size by 2060 . Therefore, maintaining a 
positive OHRQoL for this aging group should involve a public health initiative to promote all aspects of healthy living, including a healthy oral cavity. Social activities including dining and the enjoyment of consuming healthy foods may be hindered by food avoidance or modification measures due to oral discomfort, decreased oral health, and limited oral function. Consequently, quality of life, including the biopsychosocial impact on OHRQoL, should incorporate the relationship between healthy eating habits and social participation. 


\section{CHAPTER III}

\section{METHODOLOGY}

This study utilized data from the 2007-2008 wave of the National Health and Nutritional Examination Survey (NHANES). This particular dataset included key items required to operationalize the Independent and Dependent Variables unique to the current analysis. The NHANES dataset is a collection of physical examinations and personal interviews that focus on different populations and a variety of health topics (NHANES, 2008, https://www.cdc.gov/nchs/nhanes).

The 2008 sample consisted of 12,946 people between the ages of 12 and 100+ years old living in the United States. Of the 12,946 respondents, 10,149 (78.4\%) were interviewed and 9,762 (75.4\%) were examined in the mobile exam centers (MEC). Data were collected via surveys by the National Center for Health Statistics (NCHS) regarding an individual's health status, prevalence of diseases, behavioral risks, and environmental and genetic factors that may contribute to health conditions or diseases.

The dataset and surveys are provided by ICPSR, and there is no permission required to gain access to the dataset or survey information. The data collection and corresponding variables for this study were collected from individuals aged $60+$ by both survey questionnaires and physical exams. The sample population $(n=2,154)$ consists of those who responded to the survey and exams; includes both males and females with a reported mean age of $70(S d=7.05)$; and represents the civilian noninstitutionalized population living in the United States.

The general and oral health survey questionnaires were collected via interviews in the respondents' homes by trained household interviewers from NCHS 
(Appendix B). The health examination component was conducted at a mobile exam center (MEC) that consisted of one physician, two dietary interviewers, medical technologists, a phlebotomist, a computer data manager, and "health technologists" who were trained by dentists to facilitate the dental exam. The dental exam provided information regarding the oral status of each participant, including teeth present or missing, and opposing teeth and which type of opposing teeth were present, such as two natural teeth or a natural tooth and a denture tooth, or a natural tooth and a denture.

Ethical considerations were addressed in the collection of data from NCHS by removing direct identifiers that may lead to the identification of data by subjects. The data collected by NCHS cannot be used for any other purpose than statistical analysis and for reporting findings. ICPSR users are expected to adhere to these restrictions as well.

\section{Independent Variable}

The Independent Variable (IV), Oral Health Status, consists of four mutually exclusive categories. The IV represents the oral condition for each respondent and was determined by evaluating the number of functioning teeth present or absent, and if a respondent requires the use of a denture appliance. Individuals in the sample will fall into one of the four categories: $a=$ a respondent owns an upper full denture/partial denture, $b=$ a respondent owns a lower full denture/partial denture, $c=$ the respondent has at least 20 natural teeth, including posterior teeth, or $d=$ the respondent has a maximum of 16 natural teeth and missing posterior teeth (Table 1). The four mutually exclusive categories were created by using measures from the oral health survey 
questionnaire, as well as the number of posterior contacts or posterior occluding teeth present, which was assessed during the clinical exam.

The following steps were used to create the first two mutually exclusive IV attributes representing upper arch denture use and lower arch denture use, which were measured and created via the dental survey questionnaire and represent the selfreported use of a full denture or partial denture, or no full denture or partial denture required for adequate food intake and daily function (Appendix B). These two attributes individually included: $a=$ the use of either an upper arch full denture or partial denture, or $b=$ the use of a lower arch full denture or partial denture (Fig.1a). The mutually exclusive variables respectively were coded as $1=\mathrm{yes}, 2=$ no, refused $=7$, don't know $=9$ in the self-reported dental survey. Recoding was completed to create a dichotomous variable for both the upper and lower denture survey questions.

The following steps were used to create the remaining two mutually exclusive IV categories, which included: $c=$ if a respondent had at least twenty natural teeth, including posterior teeth, or $\mathrm{d}=$ if a respondent had only sixteen or fewer natural teeth, and missing posterior teeth (Fig.2a \& 2b). These two attributes were operationalized from a variable created in the NHANES dental exam section called Functional Contact Zones for each respondent. The NHANES measure of the variable called functional contact zones represented individual functional opposing pairs of teeth for each respondent. The technicians coded $=0$ if no natural upper/lower tooth pair was visually in contact, $1=$ upper/lower natural tooth pair was visually in contact, 2 =functional contact between a natural tooth opposing either a fixed or removable prosthetic (either a crowned tooth or a denture tooth), 3 =functional contact between two removable 
opposing appliances (two denture teeth), or 9=cannot assess. The present design recoded the NHANES functional contact zone variable which included: codes $0=$ no opposing natural teeth in contact, and $1=$ an upper and lower natural opposing teeth in contact, which created the final design for the remaining two IV attributes (Fig.2a \& 2b). Consequently, the analytical sample size was reduced to 1,614 (74.9\%) respondents due to missing data on key variables. The remaining $(25 \%)$ respondents may have had a mixed assembly of occluding dentition, including natural teeth, dentures, or bridge work that was not part of the key data for the IV items. The level of measurement for the independent variable, which represents the oral health condition of an individual, is a nominal variable.

\section{Dependent Variable}

The Dependent Variable (DV), Oral Comfort, is a composite measure for each respondent to evaluate the comfort and function of their overall oral health and represents one's OHRQoL score. The composite measure construct is unique for this research design; however, its factors resemble the universal GOHAI instrument discussed previously. The index includes six items selected from the self-reported oral health questionnaire section of the survey, which were related to the respondent's oral comfort and function during the last year. The questions assessed the respondent's experiences during the last year, including: "How often in the last year have you had aching anywhere in your mouth," evaluated by seven categories (1=very often, $2=$ fairly often, 3=occasionally, 4=hardly ever, 5=never, 7=refused, 9=don't know); "How often last year felt bad because of mouth pain," evaluated by seven categories (1=very often, 2 =fairly often, $3=$ occasionally, $4=$ hardly ever, $5=$ never, $7=$ refused, $9=$ 
don't know ); "How often last year had difficulty with a job because of mouth pain," evaluated by seven categories ( 1 =very often, $2=$ fairly often, $3=$ occasionally, $4=$ hardly ever, 5= never, 7=refused, 9=don't know); "How often last year was taste affected due to mouth pain," evaluated by seven categories (1=very often, $2=$ fairly often, 3=occasionally, 4=hardly ever, 5=never, 7=refused, 9=don't know); "How often last year could not eat due to mouth pain," evaluated by seven categories (1=very often, 2=fairly often, 3=occasionally, 4=hardly ever, 5=never, 7=refused, 9= don't know); "How would you rate the condition of your mouth," evaluated by seven categories (1=excellent, 2=very good, 3=good, 4=fair, 5=poor, 7=refused, 9=don't know) (Appendix B).

An index of oral comfort was created by calculating the mean score for each respondent who had three or more non-missing items resulting in a range from 6 to 30 . Lower scores represented a poor or low oral health and comfort score, and higher scores represent better oral comfort and function, which ultimately represent overall

OHRQoL scores. Construct validity was assessed using Cronbach Alpha (0.823).

\section{Data Analysis}

Univariate analysis included examining frequency distributions of the key independent variable, oral health status. Variable attributes included: use of an upper partial/denture; use of a lower of denture/partial; had twenty teeth present; had fewer than sixteen teeth present.

Bivariate analysis included two-way contingency tables (cross-tabs) to evaluate the relationship between a respondent's "self-rated oral condition" and associated clinical oral health status. Cross tabs were conducted to have a better 
understanding of the respondent's perception of their oral health condition related to the associated clinical oral health which may influence oral health related quality of life scores.

Multivariate regression was conducted using standardized coefficients from two OLS regression models in which the OHRQoL score is the dependent variable. The first model estimated the effects of the individual oral health status attributes on the OHRQoL scores. Oral health deficits, such as owning an upper or lower denture appliance or having fewer than sixteen teeth, were compared to individuals who have the optimal number of twenty teeth present. The second model added demographic controls to the first model, including household income, education, ethnicity, age, and gender to examine how demographics predict the outcome (OHRQoL), and how controlling for demographics changes the influence of the IV on the DV. Patterns of missing data were not observed in key variables. To analyze the 2008 wave of the NHANES, the Standard Package for Social Sciences (SPSS) was used. The results of the statistical analysis was considered significant if $p<0.05$. 


\section{CHAPTER IV}

RESULTS

The 2008 wave of the National Health and Nutritional Survey (NHANES) included 2,154 men and women aged 60 years or older living in the United States. As shown in Table 2, the sample was diverse. NHANES respondents were $48 \%$ men and $51 \%$ were women. The mean age at screening was $70.6(S D=7.05)$ years. The majority of participants were non-Hispanic White (54.6\%), 20\% were non-Hispanic Black, and 22.4\% were Hispanic. Thirty thousand dollars was the median annual household income level. Almost a quarter $(24.7 \%)$ of the population had a high school diploma or equivalent, while $37 \%$ completed less than $12^{\text {th }}$ grade with no diploma, including $21 \%$ who had less than a $9^{\text {th }}$ grade educational level. The remaining sample population completed some college or Associates Degree (20.4\%) or graduated from college $(17.5 \%)$. The majority of the population had health insurance coverage from either a private insurer $(53 \%)$ or Medicare $(64 \%)$, while a small portion had no health insurance coverage $(7 \%)$. 
Table 2. Demographic Characteristics from NHANES 2008 Respondents $(\mathrm{N}=2,154)$

\begin{tabular}{lllll}
\hline & $\mathrm{N}$ & $\%$ & Mean & $S D$ \\
\hline Gender & & & & \\
Female & 1140 & $51.7 \%$ & & \\
Male & 1040 & $48.3 \%$ & & \\
Age at Screening & & & 70.6 & 7.05 \\
Race & 432 & $22.4 \%$ & & \\
Hispanic & 1,243 & $54.6 \%$ & & \\
Non-Hispanic White & 384 & $20 \%$ & & \\
Non-Hispanic Black & 95 & $3 \%$ & & \\
Other & & & 30,000 & \\
Annual Household Income* & & & \\
Education Level & & & \\
$<9^{\text {th }}$ grade & 453 & $21 \%$ & \\
9th-12th Grade & 348 & $16 \%$ & \\
High School Diploma/GED & 533 & $24.7 \%$ & \\
Some College & 439 & $20.4 \%$ & \\
College Grad/Above & 377 & $17.5 \%$ & \\
Health Insurance Coverage & & & \\
Medicare & & $64 \%$ & \\
Private Insurance & & $53 \%$ & \\
No Insurance & & $7 \%$ & \\
& &
\end{tabular}

Note.* Household income is reported as median

In the first analysis a cross-tabulation was conducted to compare differences between participants' perceived oral condition and the clinical status of their oral health. The results indicate that there is a statistically significant relationship between the two variables, $\left(\chi^{2}=130.63, p<0.001\right)$. Table 3 reports the oral health attributes and associated findings, including; those who owned an upper denture $(n=225)$, those who owned a lower denture $(\mathrm{n}=665)$, and individuals who had at least $20+$ teeth that included posterior teeth $(\mathrm{n}=550)$, or individuals missing posterior teeth resulting in less than 16 teeth $(\mathrm{n}=174)$. Among those with an upper denture, 25.3\% perceived their oral health as good, while more than half perceived their oral health as fair to poor 
(49.8\%). Respondents owning a lower denture reported having poor oral health (14.3\%), while $34 \%$ reporting having very good to excellent oral health. The majority of participants $(66 \%)$ who had optimal $20+$ teeth reported having good to excellent oral health, and $17.9 \%$ reported poor oral health. The mode of participants $(41.6 \%)$ missing posterior teeth and with fewer than 16 teeth reported poor oral health, while $23 \%$ reported good oral health.

Table 3. Individual's Perceived Condition of Oral Health Based on Oral Health Status $(\mathrm{N}=1,614)$

\begin{tabular}{|c|c|c|c|c|c|c|c|}
\hline & $\begin{array}{l}\text { Poor } \\
(\mathrm{n}=296) \\
\%(\mathrm{n})\end{array}$ & $\begin{array}{c}\text { Fair } \\
(\mathrm{n}=2922) \\
\%(\mathrm{n})\end{array}$ & $\begin{array}{l}\text { Good } \\
(\mathrm{n}=470) \\
\%(\mathrm{n})\end{array}$ & $\begin{array}{l}\text { Very } \\
\text { Good } \\
(\mathrm{n}-=207) \\
\%(\mathrm{n})\end{array}$ & $\begin{array}{c}\text { Excellent } \\
(\mathrm{n}=201) \\
\%(\mathrm{n})\end{array}$ & $\begin{array}{l}\text { Chi- } \\
\text { Square }\end{array}$ & p-value \\
\hline $\begin{array}{l}\text { Upper } \\
\text { Denture }\end{array}$ & $29.6 \%(69)$ & $20.2 \%(59)$ & $25.3 \%(73)$ & $12.0 \%(14)$ & $12.5 \%(18)$ & 130.6 & $0.000^{* * * *}$ \\
\hline $\begin{array}{l}\text { Lower } \\
\text { Denture }\end{array}$ & $14.3 \%(94)$ & $18 \%(119)$ & $33.6 \%(221)$ & $14.9 \%(98)$ & $19.1 \%(126)$ & & \\
\hline $\begin{array}{l}\text { 20+Teeth/ } \\
\text { \&Posterior }\end{array}$ & $17.9 \%(61)$ & $18.9 \%(76)$ & $33.7 \%(136)$ & $19.6 \%(79)$ & $12.7 \%(51)$ & & \\
\hline $\begin{array}{l}<16 \text { Teeth/ } \\
\text { (No } \\
\text { Posteriors) }\end{array}$ & $41.6 \%(72)$ & $21.9 \%(38)$ & $23.1 \%(40)$ & $9.2 \%(16)$ & $4.2 \%(7)$ & & \\
\hline
\end{tabular}

$* p<0.05, * * p<0.01, * * * p<0.001$

Multiple linear regression models were constructed to investigate whether an individual's oral health status and demographic variables significantly predict one's OHRQoL scores. Table 4 reports standardized and unstandardized coefficients from two OLS regressions where OHRQoL scores were the dependent variable. The first model estimates the effects of oral health deficits on OHRQoL scores compared to 
OHRQoL scores for individuals who had optimal 20+ teeth present. The results indicate that oral health deficits were a significant predictor of OHRQoL scores, $\mathrm{F}(4,952)=7.242, p<0.001$. The strongest predictor was whether an individual had no posterior teeth present $(\beta=0.478, p<0.001)$. Also, an inverse relationship was identified between individuals owning a lower denture and OHRQoL $(\beta=0.339$, $p<0.001$ ). Compared to individuals with $20+$ teeth, those with a lower denture had a lower OHRQoL score. The analysis did not show a statistically significant difference in OHRQoL between owning an upper denture and the comparison group (20+ teeth present). The linear regression results show that only $3 \%$ of respondents' OHRQoL scores can be explained by oral health deficits, such missing posterior teeth or having an upper or lower denture.

The second model added a set of standard demographics and household income control variables to Model 1 . The results indicate that after controlling for oral health deficits, demographic variables were a significant predictor of OHRQoL, $\mathrm{F}(8,850)=7.710, p<0.001$. Education was the strongest demographic predictor for OHRQoL scores $(\beta=0.146, p=0.000)$. Individuals with more education have higher OHRQoL scores. Race/Ethnicity in general was not a significant predictor for OHRQoL except for the Hispanic population $(\beta=0.092, p<0.001)$. Compared to the White population, Hispanics had lower OHRQoL scores. Additionally, gender, age, and household income were not significant predictors of the dependent variable. Notably, having no posterior teeth present remains the strongest predictor for the dependent variable $(\beta=0.232, p<0.001)$, as well as owning a lower denture $(\beta=0.246$, $p<0.001)$. The linear regression results from this model show that $6 \%$ of the variance 
in the data can be explained by demographic coefficients plus oral health deficits.

Demographic variables had a significant impact on OHRQoL scores because the variance explained by the model almost doubled $\left(\mathrm{R}^{2}=0.059\right)$ after adding these variable to the regression model. Additionally, after controlling for demographic differences, the results from model 1 remain largely the same, though the effect size (Beta coefficients) are reduced.

Table 4

Linear Regression Results: OHRQoL scores by Oral Health Status and Socioeconomic Status: NHANES $2008(\mathrm{~N}=1,640)$

\begin{tabular}{|c|c|c|c|c|c|c|}
\hline \multirow[b]{2}{*}{ Variable } & \multicolumn{2}{|c|}{ Model 1} & \multicolumn{4}{|c|}{ Model 2} \\
\hline & B & SE & $\boldsymbol{\beta}$ & B & SE & $\boldsymbol{\beta}$ \\
\hline Upper Denture & -0.401 & 0.625 & 0.043 & -0.230 & 0.638 & 0.025 \\
\hline Lower Denture & -0.996 & 1.00 & $0.339 * *$ & -0.946 & 0.986 & $0.246 * *$ \\
\hline \multicolumn{7}{|l|}{ No Posterior } \\
\hline Teeth Present & -1.907 & 0.751 & $0.478 * *$ & -1.162 & 0.757 & $0.266 * *$ \\
\hline \multicolumn{7}{|l|}{ Ethnicity } \\
\hline Black & & & & -0.068 & 0.348 & 0.007 \\
\hline Hispanic & & & & -1.253 & 0.467 & $0.092 * *$ \\
\hline Other & & & & -0.132 & 0.920 & 0.005 \\
\hline \multicolumn{7}{|l|}{ Gender } \\
\hline Female & & & & 0.020 & 0.638 & 0.012 \\
\hline $\begin{array}{l}\text { House-hold } \\
\text { income }\end{array}$ & & & & 0.007 & 0.007 & 0.023 \\
\hline Education & & & & 0.485 & 0.115 & $0.146 * * *$ \\
\hline Age & & & & 0.031 & 0.021 & 0.048 \\
\hline Constant & & & 33.645 & & & 19.204 \\
\hline $\mathrm{R}^{2}$ & & & 0.030 & & & 0.068 \\
\hline Adjusted $\mathrm{R}^{2}$ & & & 0.037 & & & 0.059 \\
\hline F Change & & & 7.242 & & & 7.710 \\
\hline $\mathrm{DF}$ & & & $966 * * *$ & & & $858 * * *$ \\
\hline Number of Cases & & & 1,614 & & & 1,614 \\
\hline
\end{tabular}

$*_{p}<0.05, * * p<0.01, * * * p<0.001$

Model 1 is unadjusted for covariates- $20+$ teeth present is the comparison group Model 2 is adjusted for Demographic Controls

- Non- Hispanic White is the comparison group for race/ethnicity 
The findings of the analysis comparing individual oral health attributes and OHRQoL scores were significant; however, oral health deficits indicate a moderate to small predicator of (OHRQoL) based on the r-squared values. Additionally, participants with the optimal number of twenty teeth present had higher OHRQoL mean scores $(M=26.0, S D=3.9)$, indicating better $O H R Q 0 L$ as compared to those who had oral health deficits, including missing posterior teeth resulting in fewer than 16 teeth, having an upper denture, and having a lower denture; see Table 5.

Table 5.

OHRQoL Mean Score for Oral Health Attributes $(\mathrm{N}=1,614)$

\begin{tabular}{llcl}
\hline Variable & $\mathrm{n}$ & MEAN & SD \\
\hline $\begin{array}{l}\text { 20+ teeth/ } \\
\quad \text { Posterior }\end{array}$ & & & \\
$\quad$ Teeth Present & 550 & 26.0 & 3.9 \\
$<16$ Teeth/ & & & \\
$\quad$ No Posteriors & 174 & 23.3 & 5.2 \\
Upper Denture & 225 & 25.3 & 4.8 \\
Lower Denture & 665 & 24.4 & 4.7 \\
Total & 1,614 & 25.1 & 4.7
\end{tabular}




\section{CHAPTER V}

\section{DISCUSSION}

The findings of this study via linear regression models support the hypothesis that poorer oral health is associated with decreased OHRQoL. Many of the findings in this study are similar to those in prior studies, although prior studies identify a stronger relationship between oral health status and OHRQoL scores (Quandt et al., 2011; Spanemberg et al., 2019). One possible reason for this discrepancy may be the limited variables examining OHRQoL included in the current analysis compared to other studies, which have used a more detailed and comprehensive OHRQoL composite scale, such as the GOHAI scale. The GOHAI model includes more items in their OHRQoL scale to reflect a better understanding of oral comfort for individuals. The items included in the GOHAI survey design include: difficulty swallowing, trouble chewing fruits and vegetables, displeased with appearance, dry mouth issues, and concerned about eating in front of people (John et al., 2016). These additional items may better reflect OHRQoL scores than the limited OHRQoL scale used in the current study analysis. In other words, a full range of measures would have better reflected an individual's oral comfort; however, such measures were not available in the creation of the present OHRQoL scale. Hence, the current model represents a partial reflection of OHRQoL scores compared to the more comprehensive GOHAI model.

Several prior studies supported the elements required to maintain optimal oral comfort and function, including a full dentition, good salivary flow, good muscle strength for proper consumption of food, and social enjoyment (Savaco et al., 2008). 
In the current study, the strongest relationship with OHRQoL scores was with whether an individual had posterior teeth or no posterior teeth present. Previous research strongly supports this finding with several strong analytic studies. Savaco et al.'s (2008) study determined that individuals with fewer teeth had more difficulty eating, less enjoyment eating, and more limited food choices than those who had an optimal number of teeth. Other studies determined that individuals with missing teeth had more discomfort, more trouble eating properly, and were more concerned about their appearance (Gotfredsen \& Walls, 2007; Rodrigues et al., 2009).

Interestingly, the current study found a significant relationship between lower denture use and OHRQoL scores compared to individuals with 20+ teeth present. Arguably, similar studies strongly support that upper and lower denture use significantly contributes to poor oral comfort and function. Lucena et al.'s (2011) study reported that $39 \%$ of older adults were dissatisfied with their dentures, and denture use negatively impacted the participant's quality of living, especially eating in general, with lower denture use found to be most debilitating. In contrast, the oral health deficit of having an upper denture was a non-significant contribution to OHRQoL compared to individuals with 20+ teeth. Additionally, having an upper denture was a non-significant contribution to OHRQoL after demographic controls were added to the second regression model as well. The explanations for an upper denture appliance having a non-significant relationship in this analysis remain unclear. A possible explanation for the non-significant findings in the current study may be representative of the NHANES data collection, which combined those who had a full denture and those who had a partial denture. A full denture and a partial denture are 
not the same type of appliance, do not function the same way, and have different outcomes (Cousson et al., 2011). Generally, an upper partial denture provides an individual with more support and comfort compared to a full upper denture (Lucena et al., 2011). The current analysis could not separate these two distinct attributes, which may have resulted in a higher OHRQoL score for this group due to the positive influence of an upper partial denture appliance. Additionally, combining these mutually exclusive variables may have influenced the non-significant finding for respondents with an upper denture appliance compared to those who had an adequate dentition of 20+ teeth. Finally, the current study found an inverse relationship between lower denture use and OHRQoL scores compared to those individuals with optimal 20+ teeth. Many studies support the current findings regarding lower denture dissatisfaction (Bessadet et al., 2013: Prithviraja et al., 2014). Moreover, Walls \& Steele's (2004) study reported that eating can cause instability with lower denture use and individuals must learn how to cope and compensate for such instability.

Furthermore, the present survey design used questions that were generalized and may not have reflected the participant's accurate oral comfort and function, due to the participant's perception and interpretation of each specific question. Also, research reports that individuals slowly compensate and modify food behavior and daily activities based on oral comfort and function (Quandt et al., 2009). Additionally, these modifications and adjustments are slow and gradual over time, with individuals unaware of their own compensating behavior (Savaco et al., 2010). Therefore, the responses to several survey questions from the NHANES study may reflect an individual's already adjusted behavior rather than a more precise description of 
present OHRQoL scores. One example of a marginal survey question was, "did you have any trouble eating during the past year?" Quandt et al.'s (2009) study that was previously mentioned, reports that individuals slowly modify eating habits based on comfort, and may be unaware of these changes or restrictions. Perhaps some of the individual OHRQoL scores would have been lower if the nutritional survey questions were more focused and included categories of foods similar to the GOHAI model. Another variable that may have altered the results was, "did you avoid work due to mouth pain this year?" The average age of respondents in the current analysis was $60+$ years old, which may not reflect those who were working. Therefore, this question may not be appropriate for this age group to acquire accurate data. The overall results in this analysis indicated a rather inflated OHRQoL score, $\mathrm{M}=25(S D=4.04)$, where six is the lowest possible score representing poor OHRQoL and thirty is the highest. Hence, the overall OHRQoL scores may reflect the quality of the question rather than the accuracy of the data, and may have lowered the confidence in the current composite measure. Finally, the survey questions included any experiences that occurred over a one-year time period. Studies suggest that it may be difficult for an older adult to recall all experiences that have transpired over a lengthy time span (Swarthout, Holly, Newton, 2019).

Respondents' perceptions and interpretations were also observed in the cross tab results that compared clinical oral health status and an individual's perceived condition of oral health. On average, participants perceived their overall oral health as good. Interestingly, a number of individuals owning a lower denture considered their oral health as excellent, and a number of individuals with a full dentition that included 
$20+$ teeth present reported poor oral health. The previous findings reflected individual interpretation that may have contributed to the overall OHRQoL scores, and possibly inflated such scores for individuals who owned a lower denture and considered their oral health as excellent. Notably, Hernadez et al.'s (2015) study determined that one's perceived oral health can influence OHRQoL evaluation outcomes. Additionally, several studies report the difficulties denture users have regarding comfort and function, especially lower denture users (Prithviraja et al., 2014). Arguably, the current findings' cross tabulation results suggest that many individuals having a lower denture indicated their oral health to be excellent, which may reflect one's oral health literacy. Therefore, OHRQoL scores may have reflected one's already modified oral function rather than accurate clinical oral function in this study.

Finally, demographic characteristics, including ethnicity and education, were strong indicators of OHRQoL scores. The incidence of poor oral health, tooth loss, and edentulism is more prevalent in minority elders, including African American and Hispanic older adults (Wu et al., 2014). Poor oral health may reflect the oral health disparities present in certain populations, as well as limited access to dental care in this population due to one's socioeconomic status. However, the current study found that the Hispanic ethnic group had a significant association with the dependent variable, compared to the Black population with no significant relationship. Interestingly, household income was found to be a non-significant indicator for OHRQoL scores. Similar studies report that dietary habits influenced by oral health are affected by affordability and accessibility of dental care (Andrade et al., 2009). Additionally, National Aging Reports indicate that $30 \%$ of the older adult population living in the 
US have many unmet needs due to economic constraints; these unmet needs will affect their oral health status and ultimately their quality of life (Lamster \& Northridge, 2008). Contrary to previous findings, income did not impact overall OHRQoL scores in the current analysis. Furthermore, the current analysis found education to be a strong predicator of the dependent variable. Educational levels were associated with a positive influence on OHRQoL in the current analysis. Hernandez et al.'s (2015) study reported poor oral health was associated with sociodemographic conditions, such as lower educational levels and lower income levels. The remaining sociodemographic variables in this study, including age and gender, were not significantly associated with OHRQoL. Notably, the respondents in the current analysis have a median household income level of $\$ 30,000.00$ annually, and more than half of the respondents (57\%) had less than a high school diploma in the current study.

Studies report that lower OHRQoL impacts social connections, and poorer oral health may have a negative impact on overall quality of life (Spamberg et al., 2019). Additionally, poor oral health may restrict chewing efficiency, lower self-esteem, and decrease social interactions (Griffin et al., 2012). Furthermore, social participation, healthy eating, and daily physical activities, representing the biopsychosocial model, are strongly correlated with one's OHRQoL (Ferreira et al., 2017). The same study reports that healthy eating and social connections (including dining out) are important determinants of older adults maintaining quality of life (Ferreira et al., 2017). Finally, socio-behavioral determinants of OHRQoL, including social activities, are influenced by oral health, function, and comfort, which are largely determined by access to dental care to prevent oral health deficits (Spanemberg et al., 2017). 
As a dental hygienist, with many years in a periodontal setting and working with older adults, I have never had an experience with a patient who was pleased with a denture appliance, never had a patient who thought their dentures were effective, and never had a patient report that they had negligible restrictions due to such appliances. In fact, the majority of my patients owning a denture appliance thought that it impacted their daily life on a large scale. Additionally, the same patients generally did not utilize a denture appliance due to the discomfort it caused and the challenges encountered with consuming food. Most denture patients expressed the desire to have their own natural teeth again!

\section{Limitations}

The present study has several limitations that may have impacted the confidence and reliability of the OHRQoL scores, as well as the overall results. First, the structure of survey questions was non-specific, which may have resulted in the respondents' perceptions and interpretations due to the framework of the survey design. John et al.'s (2016) study reports that an OHRQoL index should include items related to oral function, oral pain, appearance, and psychosocial impacts collectively. More focused and tailored questions might have resulted in more accurate data to evaluate and understand the impact that poorer oral health has on OHRQoL.

Second, the NHANES 2008 wave collection of data made it challenging to create mutually exclusive variables to evaluate. The data collection for a respondent having an upper denture or lower denture was operationalized with respondents having an upper partial denture or lower partial denture collectively. A denture and a partial denture are not the same type of appliance, as they individually function differently 
and have different outcomes for the owners of such appliances (Bassadet et al., 2013). Therefore, these variables are mutually exclusive and should have been operationalized independently via the survey design; however, the NHANES collected this data together, and they could not be separated for individual analysis.

Finally, the NHANES 2008 wave used trained medical assistants for clinical data collection, including evaluating the number of teeth missing for each respondent, presence of occluding teeth or non-occluding teeth, and restorative work present or not (NHANES, 2008, https://www.cdc.gov/nchs/nhanes). The training, time spent on training, and evaluation for effective training methods was unclear. Interestingly, the NHANES 2015 wave utilized dentists for the oral health data collection module and not trained medical assistants (NHANES, 2015, https://www.cdc.gov/nchs/nhanes).

The current analytical study had several strengths. First, the sample population was large with an equal distribution of men and women. Second, the population was diverse and included a random selection of community-dwelling older adults living in the US. Additionally, the NHANES 2008 wave dataset provided comprehensive and detailed items for the current analytical study, including key variables. Finally, the current analysis provides future direction for public policies to address the burden of oral disease that can negatively affect the biopsychosocial relationship in the older adult population.

\section{Future Directions}

Future investigations between oral health indicators and OHRQoL will assist in creating a clearer understanding of how OHRQoL impacts social outcomes in older adults, as well as improving healthy aging for this group via maintaining good oral 
health. Many policies focus on the physical needs of older adults and exclude the biopsychosocial needs of this population via quality of life indicators (Douglas et al., 2017) In other words, it would be beneficial for public policies to incorporate a full scope of indicators to improve healthy living for older adults, including social inclusion, optimal oral health, physical health, and financial stability. The Older Americans Act supports funds for food acquisition for the older adult population, with such programs as "Meals on Wheels" (Lamster \& Northridge, 2008). However, policies should also address how such food is being consumed and how well individuals are consuming healthy foods. Quandt et al.'s (2009) study reports that there has been little research conducted to investigate the consequences of oral health deficits on eating habits in older adults. Additionally, policies should address what, if any, oral health deficits are present that may contribute to unintentional social isolation due to oral pain or insufficiencies, as well as to food modification. Similar studies indicate that community programs that address oral health deficits can promote healthy living for older adults (Griffin et al., 2012). In other words, public health policies need to address how well older adults are functioning in their daily lives, including the influence of poor oral health on food consumption, as well as such social connections as dining with others.

The biomedical model is also important to address, including clinical oral health indicators for optimal oral health, as well as using the biopsychosocial model for optimal OHRQoL (Spanemberg et al., 2017). Oral health deficits are an example of the clinical indicators representing the oral health needs and access to dental care concerns that impact older adults and negatively influence biopsychosocial indicators, 
such as social connections facilitating emotional well-being (Spanemberg et al., 2017). Consequently, older adults face more health inequalities, including dental care accessibility and dental insurance coverage compared to other age groups (Lawrence, 2016). Medicare does not cover dental needs, and many older adults do not have dental insurance to facilitate proper dental care (Roehr, 2008). This burden for older adults in the US requires timely consideration due to the increase in life expectancy of this population that is the fastest growing age group according to the US Census (Elder Care Report, 2020). Additionally, the same report indicates the number of older adults $65+$ will be greater than individuals under the age of eighteen by 2035 (Elder Care Report, 2020). Finally, oral health disparities have improved over the decades; however, older adults and people who fall below the US poverty line have not seen such an oral health improvement (Elder Care Report, 2020).

Ultimately, future policy directions should address ways to enhance social participation via oral health indicators to comprehensively promote and support healthy aging. Dental disease is mostly preventable, and aging alone is not a precursor for oral health deficits (Griffin et al., 2012). Moreover, ageism or the physical decline in age is viewed as a normal process in the older adult (Moody \& Sasser, 2012). However, oral health deficits in the older adult population represent the accumulation of poor dental care over the life course of an individual rather than a normal part of aging (Griffin et al., 2012). The additional challenge for policymakers will be the inclusion of the older adult population in considerations of oral health disparities and ultimately addressing such disparities for this forgotten but deserving population. The focus of OHRQoL is a paradigm shift from quantity of life to quality of life for the 
older adult population. Many countries have already adopted this paradigm shift in the quality over quantity of life philosophy, such as Japan. Japan is considered the superpower for caring for their elders, as well as the country that has the highest number of centenarians (Arai et al., 2013). Japan's philosophy is to maintain quality of life and healthy living for their older adult population for as long as possible, rather than merely sustaining life (Arai et al., 2013). Forward thinking policies for older adults in the US should reflect this paradigm shift that focuses on quality of life, which will ultimately create and facilitate healthy living, including OHRQoL, for the older adult population as long as possible. 


\section{APPENDIX A}

\section{Glossary of Terms}

Malnutrition- Lack of proper nutrients caused by not having enough to eat, not eating the right nutrients, or inability to use the food that one does eat. US statistics report one in every two older adults is malnourished or at risk for malnutrition.

Polypharmacology- The design or use of pharmaceutic agents that act on multiple targets in the body or on the disease pathway.

Functioning dentition- Having 20 or more teeth present or ten occluding pairs of teeth (upper arch and lower arch).

Maxillary- Refers to the upper jaw.

Mandible- Refers to the lower jaw.

Sarcopenia- The degenerative loss of skeletal muscle. In the context, it is referring to lip and tongue muscles.

Masticatory function- The process by which food is crushed and ground by teeth. It is the first step in the digestive process.

Edentulism/Edentulous- Complete loss of all natural teeth.

Partial Edentulism- Loss of some, but not all, natural teeth. 
Craniofacial complex-Comprises the head, face, and oral cavity, including the maxilla and mandible in the oral cavity.

Complete Denture- Known as false teeth. Prosthetic removable device used to replace all missing teeth in the maxilla or mandible. This device is retained by maxillary bone or mandible bone by suction to remain in place.

Partial Denture- Removable prosthetic device used to replace one or more missing teeth in the maxilla or mandible. This device is held in place by surrounding teeth still remaining in the oral cavity.

Prosthetics (dental)- Oral device used to restore oral function, such as partial or full dentures, crowns, and bridge work.

Oral appliance- General term describing a full denture or partial denture prosthetic device.

Oral Cavity- Includes part of the mouth behind the gingiva, teeth that are bound above by the hard and soft palate, and teeth below by the tongue and tissues connecting to the mandible.

Oral Dysphagia- Difficulty with feeding or swallowing. It is a symptom and not a disease.

Xerostomia- Known as a dry mouth, resulting from reduced or absent saliva. It is a symptom and not a disease that is often caused by medications. 


\section{Appendix B}

NHANES Survey Questions (via interview)

Do you own a maxillary (upper) denture or partial denture?

$1=$ yes $\quad 2=$ no

Do you own a mandibular (lower) denture or partial denture?

$1=$ yes $\quad 2=$ no

Do you usually wear a maxillary (upper) denture or partial denture during the day?

$1=$ yes $\quad 2=$ no $\quad 3=$ refuse $4=$ don't know

Do you usually wear a mandibular (lower) denture or partial denture during the day?

$1=$ yes $\quad 2=$ no $\quad 3=$ refuse $4=$ don't know

How would you rate your overall health condition?

$1=$ excellent $2=$ very good $3=$ good $4=$ fair $5=$ poor $7=$ refuse $9=$ don't know 8

Do you have health insurance coverage?

$1=$ yes $\quad 2=$ no $\quad 7=$ refuse $\quad 9=$ don't know

How would you rate the condition of your mouth?

$1=$ excellent $2=$ very good $3=$ good $4=$ fair $5=$ poor $7=$ refuse $9=$ don't know

How often in the last year have you had aching anywhere in the mouth?

$1=$ very often 2 = fairly often $3=$ occasionally $4=$ hardly ever $5=$ never $7=$ refuse $9=$ don't know

How often in the last year felt bad because of mouth pain?

Same scale as above

How often in the last year had difficulty with job because of mouth pain?

Same scale as above 
How often in the last year taste was affected due to mouth pain?

Same scale as above

How often in the last year could not eat due to mouth pain?

Same scale as above

How often in the last year felt embarrassed due to mouth?

Same scale as above

Gender

$1=$ male $2=$ female

Age at screening

$18 \mathrm{yrs}-150 \mathrm{yrs}$

What is your race/ethnicity?

1=Mexican American 2=Hispanic 3=white 4=black 5=other

Education level at screening

$1=$ less then $9^{\text {th }}$ grade $2=9^{\text {th }}-12^{\text {th }}$ grade $3=$ high school or GED $4=$ some college $5=$ college/beyond

Marital status at screening

$1=$ married $2=$ widowed $3=$ divorced $4=$ separated $5=$ living with partner $6=$ never married 
Figure 1a. (upper denture and lower denture appliances)

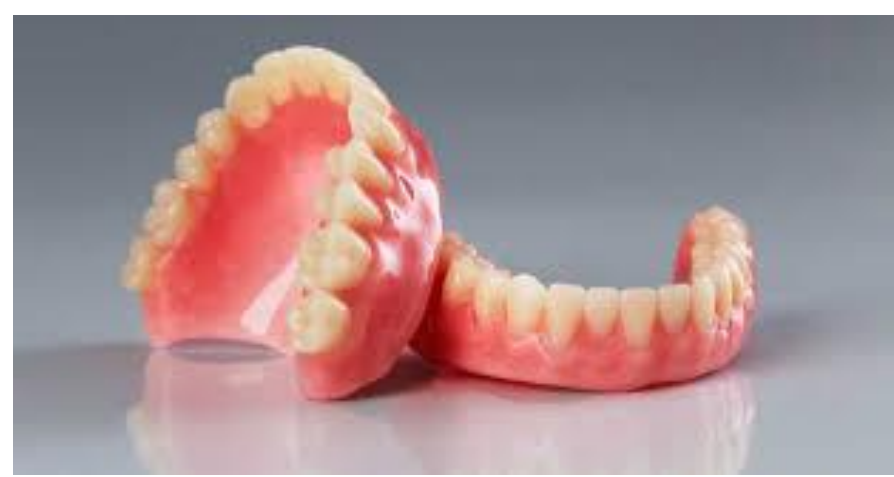

Figure 1b. (partial denture and full denture appliance)

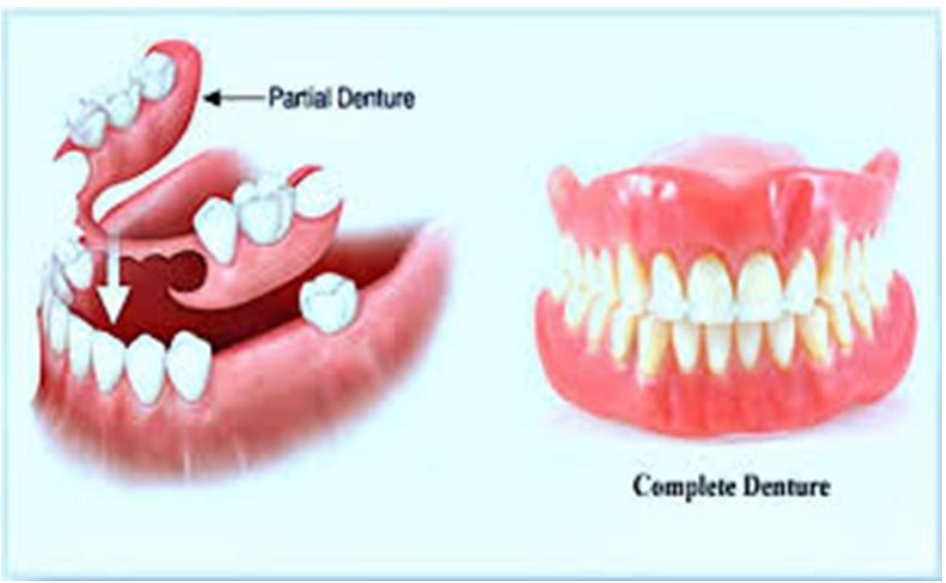


Figure 2 a. Functioning dentition $=$ At least $20+$ teeth, including posterior teeth

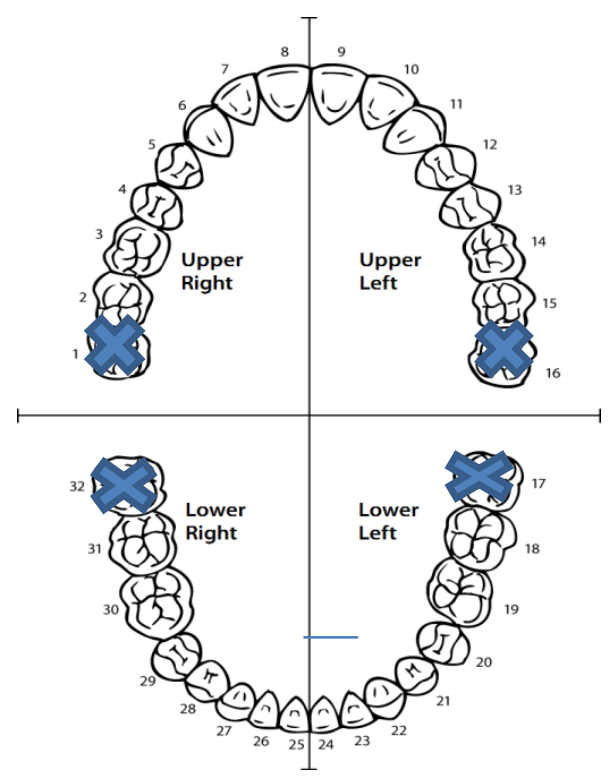

Figure $2 b$. Non-functioning dentition $<16$ teeth, and no posterior teeth

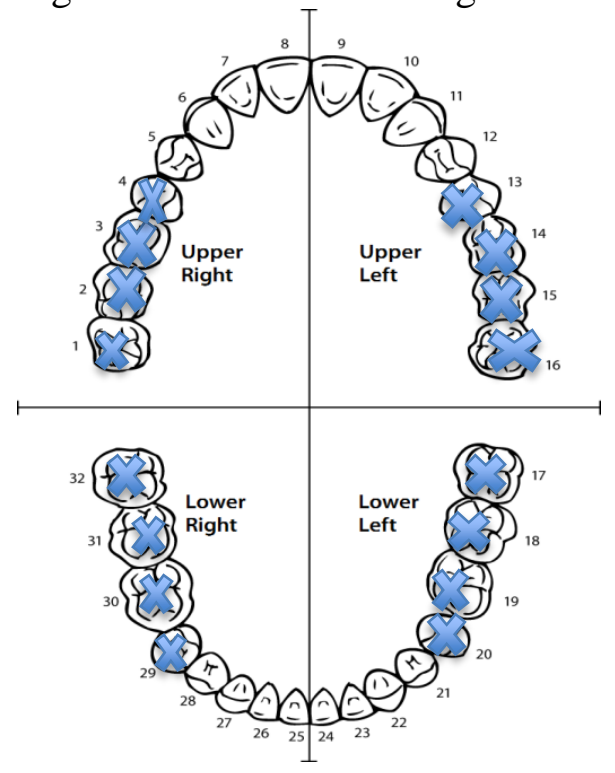


Table 1.

Independent Variable Attribute Table

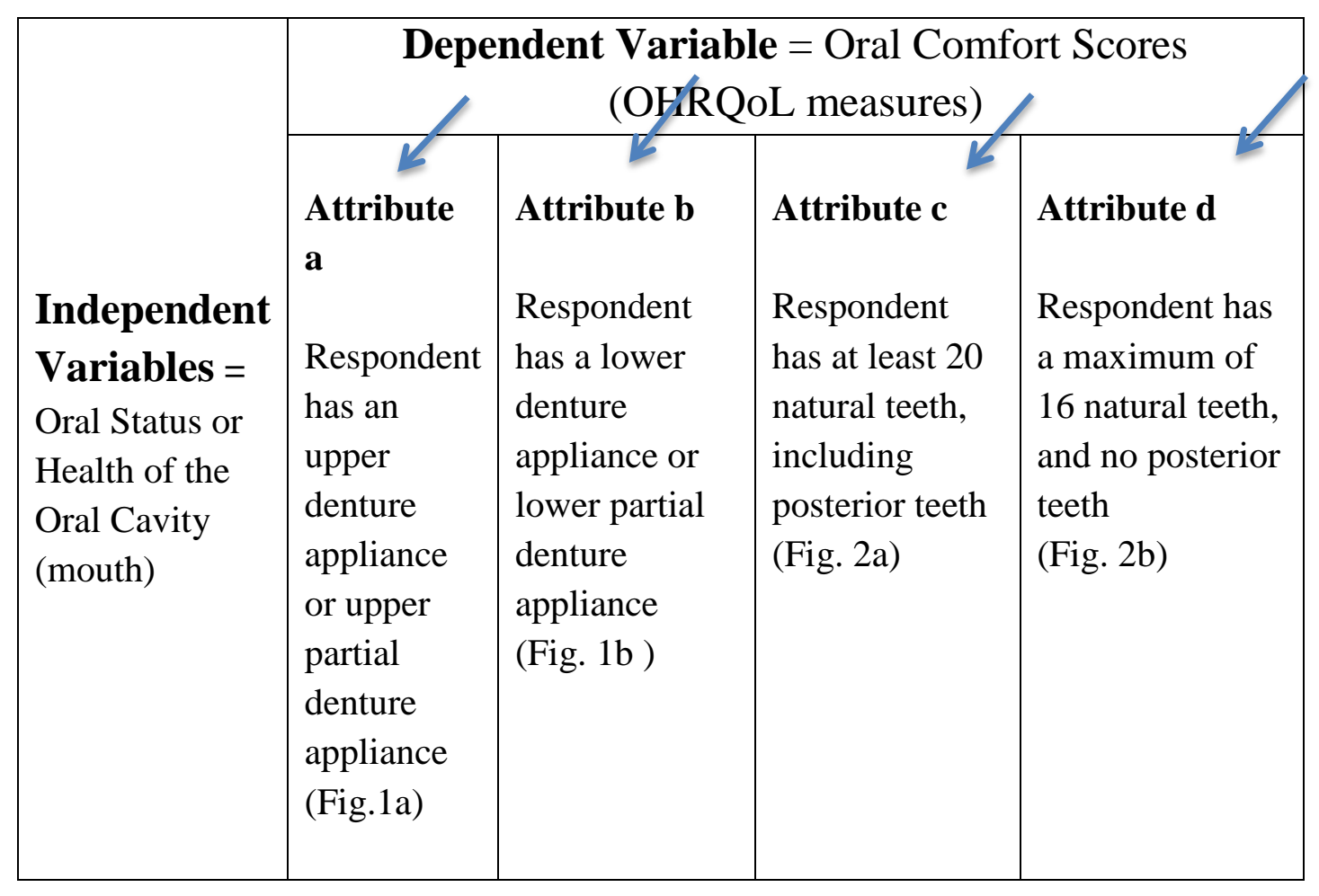




\section{BIBLOGRAPHY}

Arai, h., Ouchi, Y., Toba, K., Shimokoda, K., Endo, T., Tsubota, K., Matsou, S., Mori, H., Yumura, W., Yokod, M., Rakugi, H., Ohshima, S. (2015). Japan as the Front- Runner of Super-Aged Societies: Perspectives from Medicine and Medical Care in Japan. Geriatric Gerontology Int. 15, 673-687. doi:

$10.1111 /$ ggi. 12450

Bassadet, M., Nicolas, E., Sochat, M., Hennequin, M., Veyrune, J.L. (2013). Impact of Removable Partial Denture Prosthesis on Chewing Efficiency. Journal of Applied Oral Science, 21(5), 392-396. doi: 10.1590/1679-775720130046

Benndi, D., Reddy, C.V. K. (2013). Oral Health Related Quality of Life. Journal of Int. Soc. Prevent Community Dental, 3(1), 4-6. doi: 10.4103/22310762.115700

Cousson, P.V., Bessadet, M., Nicolas, E., Veyrune, J., L., Lesourd, B., Lassauzay, C. (2011). Nutritional Status, Dietary Intake and Oral Quality of Life in Elderly Complete Denture Wearers. Gerontology Society, 29, 685-692. doi:10.1111/j.1741-2358.2011.00545x

Douglas, H., Georgiou, A., Westbrook, J. (2017). Social Participation as an Indicator of Successful Aging: An Overview of Concepts and Their Association with Health. Australian Health Review, 41, 455-462. doi: 10.1071/AH16038

Eldercare Report. Legislative, Health, Governance, and Related Matters (2020), Elder Care Workgroup Report (13). Retrieved on January 10, 2021, from https://ncea.acl.gov/What-We-Do/Policy/Government-Reports.aspx 
Emami, E., De Sousa, R.F., Kabawat, M., Feine, J.S. (2013). The Impact of Edentulism on Oral and General Health. International Journal of Dentistry. Epub May, 2013. PMID: 2373789. doi: 10.1155/2013/498305

Favaro-Moreia, N.C., Krausch-Hofmann, S., Matthys, C., Vereecken, C., Vanhauwaert, E.,Declercq, A., Berring, G.E., Duyck, J. (2016). Risk Factors for Malnutrition in Older Adults: A Systemic Review of the Literature Based Longitudinal Data. Advances in Nutrition, International Review Journal, 7(3), 507-522. doi: 10.3945/an.115.011254

Ferreira, M.C.G., Tura, L.F.R., Da Silva, R.C., Ferreira, M.D. (2017). Social Representation of Older Adults Regarding Quality of Life. Good Practices: Fundamentals of Care in Geriatric Nursing, 70(4), 806-813. doi: 10.1590/0034-7167-2017-0097

Griffin, S.O., Jones, J.A., Brunson, D., Griffin, P., Bailey, W.D. (2012). Burden of Oral Disease among Older Adults and Implications for Public Health Priorities. American Journal of Public Health, 102(3), 411-418. doi: 10.2105/AJPH.2011.300362

Gotfredsen, K., Walls, Angus W. (2007). What Dentition Assures Oral Health, Clinic Oral Impl Res, 18 (3), 34-45. doi:10.1111/j.1600-0501.01436.x

Hernandez, R.D., Ramirez, V., Jarillo, E.C., Irioyen, M.E., Mendoza, V.M. (2015). Relationship between Gender, Income and Education and Self-Perceived Oral Health among Elderly Mexicans. An Exploratory Study. Cienc. Saude Colectiva, 20 (4). Retrieved on March 1, 2021, from https//www.scielo.br/scielo. doi: 10.1590/1413-81232015204.0070214 
Hummel, S. K., Wilson, M. A., Marker, Victoria, A., Nunn, Martha, E. (2002).Quality of Removable Partial Dentures Worn by the Adult U.S. Population. Journal of Prosthetic Dentistry, 88(1), 37-43 doi:10.1067/mpr.2002.126845

John, M., T., Reissman, D.R., Celebic, A., Baba, K., Kende, D., Larsson, P., Rener-Sitar, K. (2016). Intergration of Oral Health-Related Quality of Life Measures. Journal of Dentistry, 53, 38-43. doi: 10.1016/j.jdent.2016.06.006

Kossioni, A. (2018). The Association of Poor Oral Health Parameters with Malnutrition in Older Adults: A Review Considering the Potential Implications for Cognitive Impairment. Nutrients, 10(11). e1709. doi: 10.3390/nu10111709

Kum, S., Northridge, M.E., Metcalf, S.S. (2018). Using Focus Groups to Design Systems Science Models That Promote Oral Health Equalities. Biomed Central Oral Health, 18(1), 99. PMID: 29866084. doi: 10.1186/s12903-018-0560-0

Lamster, I., Northridge, M. (2008). Improving Oral Health for the Elderly: An Interdisciplinary Approach. New York, NY. Springer Science and Business Media

Lawrence, A. (2016). Aging in America: An Agenda for an Era of New Possibilities. Journal of the American Society on Aging, 40(4), 6-9.

Lucena, S.C., Gomes, S.G.F., Da Silva, W., Del Bel, Cury, A.A. (2011). Patients' Satisfaction and Functional Assessment of Existing Complete Dentures: Correlation with Objective Mastication. Journal of Oral Rehabilitation, 38, 440-446. doi: 10.1111/j.1365-2842.2010.02174.x 
Moody, H.R., Sasser, J.R. (2021). Aging: Concepts and Controversies, $10^{\text {th }}$ Edition Thousand Oaks, CA. SAGE Publications, Inc.

National Aging Report (2020). Retrieved December, 29, 2020, from https://www.n4a.org/2017aaasurvey

National Health and Nutritional Examination Survey (NHANES) (2008). Retrieved April, 1, 2019 from https://www.cdc.gov/nchs/nhanes/index.htm.

National Health and Nutritional Examination Survey (NHANES) (2015). Retrieved

December, 1, 2020 from https://www.cdc.gov/nchs/nhanes/index.htm.

Prithviraj, D.R., Madan, V., Harshmayi, P., Kumar, C.G., Vashisht, R. (2020). A Comparison of Mastication Efficiency in Conventional Dentures, Implant Retained or Supported Over- Dentures and Implant Supported fixed Prosthesis: A Literature Review. Journal of Dental Implants, 4(2), 153-157

Quandt, S. A., Savoca, M., R., Leng, X., Chen, H., Bell, R.A., Gilbert, G.H., Anderson, A., M., Kohrman, T., Acury, T. A. (2011). Dry Mouth and Dietary Quality in Older Adults in North Carolina. Journal of American Geriatrics Society, 59, 439-445. doi: 10.111/j.1532-5415.2010.03309.x

Quandt, S. A., Chen, H., Bell, R.A., Savoca, M. R., Anderson, A. M., Leng, X., Kohrman, T., Gilbert, G.H., Acury, Thomas, A. (2009). Food Avoidance and Food Modification Practices of Older Rural Adults: Association with Oral Health Status and Implications for Service Provision. The Gerontologist, 50(1), 100-111. 
Rodrigues, H.L., Scelza, M.F.Z., Boaventura, G.T., Custodio, S.M., Moreira, E.A.M., Oliveira, D.L. (2010). Relation between Oral Health and Nutritional Condition in the Elderly. Journal of Applied Oral Science, 20(1), 38-44. doi: 10.1590/s1678-77572012000100008

Roehr, B. (2008). US is Urged to Expand it Healthcare Workforce to Cope with Surge in Number of Elderly People. BMJ, 333(7649). Retrieved March 20, 2020 from Medline/PubMed. PMID: 18420686 doi:10.1136/bmj.39553.682685.94

Sakai, K., Nakayama, E., Tohara, H., Kodoma, K., Ueda, K. (2017). Relationship Between Tongue Strength, Lip Strength, and Nutrition- Related Sarcopenia in Older Rehabilitation Inpatients: A Cross-Sectional Study. Clinical Interv Aging, 12, 1207-1214. doi:10.2147/cia.s141148

Savoca, M. R., Arcury, T. A., Leng, X., Chen, H., Bell, R. A., Anderson, A. M., Kohrman, T., Frazier, R. J., Gilbert, G. H., Quandt, S.A. (2009). Severe Tooth Loss in Older Adults as a Key Indicator of Compromised Dietary Quality. Health Nutrition, 13(4), 466-477. doi: 10.1017/s1368980009991236

Savoca, M. R., Arcury, T., A., Leng, X., Chen, H., Bell, R. A., Anderson, A.M., Kohrman, T., Frazier, R., J., Gilbert, G. H., Quandt, S.A. (2010). Association between Dietary Quality of Rural Older Adults and Self-Reported Food Avoidance and Food Modifications Due to Oral Health Problems. Journal American Geriatrics Society, 58(7), 1225-1232.

doi:10.1111/j.1532.5415.2010.02909.x 
Sischo, L., Broder, H.L. (2011). Oral Health Related Quality of Life: What, Why, How, and Future Implications. Journal of Dental Research, 90(11), 12641270. doi: $10.1177 / 0022034511399918$

Spanemberg, J.C., Cardosa, J.A., Slob, E.M.G.B., Lopez, J. (2019). Quality of Life Related to Oral Health and its Impact in Adults. Journal of Maxillofacial Surgery, 120, 234-239. doi: 10.1016/j.jormas.2019.02.004

Swarthout, K., Holly, J., Newton, S. (2019). Effects of Mastication on Cognitive Health. Dimensions. Retreived December 1, 2020 from www.dimensionsofdentalhygiene.com

Tiwari, T., Scarbo, S., Bryant, L.L., Puma, J. (2017). Factors Associated with Tooth Loss in Older Adults in Rural Colorado. Journal of Community Health, 41(3), 476-481. doi: 10.1007/s10900-015-0117-y

Toniazzo, M.P., Amorim, P.S., Muniz, F.W.M.G., Weidlich, P. (2018). Relationship of Nutritional Status and Oral Health in Elderly: Systematic Review with Meta-Analysis. Clinical Nutrition, 38, 824-830.

doi: 10.1016/j.clnu.2017.03.014

Walls, A.W.G., Steele, J.G., Wu, L.L., Cheung, K.Y., Lam, P.Y.P. (2004). The Relationship Between Oral Health and Nutrition in Older People. Mechanisms of Aging and Development, 125, 853-857. doi: 10.1016/j.mad.2004.07.011

Wakabayashi, H., Matsushima, M., Ichikawa, H., Murayama, S., Yoshida, S., Kaneko, M., Mutai, R. (2018). Occlusal Support, Dysphagia, Malnutrition and Activities of Daily Living in Aged Individuals Needing Long-Term Care: A 
Path Analysis. Journal of Nutrition Health and Aging, 22(1), 53-58. doi: 10.1007/s12603-017-0897-0.

Wu, L.L., Cheung, K.Y., Lam, P.Y.P., Gao, X., L. (2018). Oral Health Indicators for Risk of Malnutrition in Elders. Journal of Health and Aging, 22(2), 254-261. doi: 10.1007/s12603-017-08887-2

Wu, B., Liang, J., Plasman, B., Remle, R.C., Luo, X. (2014). Edentulism Trends among Middle-aged and Older Adults in the United States: Comparison of Five Racial/Ethnic Groups. Community Dental Oral Epidemiology, 40(2), 145153. doi: $10.1111 / j .1600-0528.2011 .00640 . x$ 